\title{
Early mobilization versus plaster immobilization of simple elbow dislocations: a cost analysis of the FuncSiE multicenter randomized clinical trial
}

\author{
Esther M. M. Van Lieshout ${ }^{1}$. Gijs I. T. Iordens ${ }^{1} \cdot$ Suzanne Polinder ${ }^{2} \cdot$ Denise Eygendaal $^{3} \cdot$ Michael H. J. Verhofstad $^{1}$. \\ Niels W. L. Schep ${ }^{4}$. Dennis Den Hartog ${ }^{1}$ on behalf of FuncSiE Trial Investigators
}

Received: 29 August 2019 / Published online: 23 November 2019

(c) The Author(s) 2019

\begin{abstract}
Introduction The primary aim was to assess and compare the total costs (direct health care costs and indirect costs due to loss of production) after early mobilization versus plaster immobilization in patients with a simple elbow dislocation. It was hypothesized that early mobilization would not lead to higher direct and indirect costs.

Materials and methods This study used data of a multicenter randomized clinical trial (FuncSiE trial). From August 25, 2009 until September 18, 2012, 100 adult patients with a simple elbow dislocation were recruited and randomized to early mobilization (immediate motion exercises; $n=48)$ or 3 weeks plaster immobilization $(n=52)$. Patients completed questionnaires on health-related quality of life [EuroQoL-5D (EQ-5D) and Short Form-36 (SF-36 PCS and SF-36 MCS)], health care use, and work absence. Follow-up was 1 year. Primary outcome were the total costs at 1 year. Analysis was by intention to treat. Results There were no significant differences in EQ-5D, SF-36 PCS, and SF-36 MCS between the two groups. Mean total costs per patient were $€ 3624$ in the early mobilization group versus $€ 7072$ in the plaster group $(p=0.094)$. Shorter work absenteeism in the early mobilization group (10 versus 18 days; $p=0.027$ ) did not lead to significantly lower costs for loss of productivity ( $€ 1719$ in the early mobilization group versus $€ 4589 ; p=0.120$ ).

Conclusion From a clinical and a socio-economic point of view, early mobilization should be the treatment of choice for a simple elbow dislocation. Plaster immobilization has inferior results at almost double the cost.
\end{abstract}

Keywords Cost-effectiveness $\cdot$ Cost utility $\cdot$ Elbow dislocation $\cdot$ Function $\cdot$ Quality of life

The members of FuncSiE Trial Investigators are listed in Acknowledgements.

Esther M. M. Van Lieshout and Gijs I. T. Iordens contributed equally.

Dennis Den Hartog

d.denhartog@erasmusmc.nl

Esther M. M. Van Lieshout

e.vanlieshout@erasmusmc.nl

Gijs I. T. Iordens

g.iordens@erasmusmc.nl

Suzanne Polinder

s.polinder@erasmusmc.nl

Denise Eygendaal

deygendaal@amphia.nl

Michael H. J. Verhofstad

m.verhofstad@erasmusmc.nl

Niels W. L. Schep

schepn@maasstadziekenhuis.nl
FuncSiE Trial Investigators

onderzoeksbureauheelkunde@erasmusmc.nl

1 Trauma Research Unit, Department of Surgery, Erasmus MC, University Medical Center Rotterdam, P.O. Box 2040, 3000 CA Rotterdam, The Netherlands

2 Department of Public Health, Erasmus MC, University Medical Center Rotterdam, P.O. Box 2040, 3000 CA Rotterdam, The Netherlands

3 Upper Limb Unit, Department of Orthopaedic Surgery, Amphia Hospital, P.O. Box 90158, 4800 RK Breda, The Netherlands

4 Trauma Unit, Department of Surgery, Academic Medical Center, P.O. Box 22660, 1100 DD Amsterdam, The Netherlands 


\section{Introduction}

The elbow is the second most commonly dislocated joint in adults and mostly occurs in young and active persons, thus affecting the working population [1-3]. A simple elbow dislocation (no associated fractures) is a disabling injury which causes considerable pain and loss of range of motion in the short term, which impedes the ability to perform daily activities such as work [4].

Previous studies suggested that early mobilization may give superior functional results [5-11]. The FuncSiE trial compared clinical outcome of early mobilization and plaster immobilization in patients with a simple elbow dislocation. The results of this study showed that early mobilization resulted in earlier recovery of elbow function and work resumption [12].

These results justify the design of a treatment guideline advocating early mobilization from a clinical point of view. However, there are no high-quality studies that report the burden of simple elbow dislocations on direct and indirect health care costs, let alone to what extent early mobilization would be able to reduce these costs. We performed a cost analysis of the FuncSiE randomized controlled trial to assess the direct and indirect costs and the cost-effectiveness of early mobilization versus plaster immobilization in patients with a simple elbow dislocation. It was hypothesized that early mobilization would not lead to higher costs.

\section{Materials and methods}

\section{Settings and participants}

This cost analysis used data of a multicenter randomized clinical trial comparing early mobilization with plaster immobilization in patients after a simple elbow dislocation (FuncSiE trial). The trial is registered at the Netherlands Trial Register (NTR2025). The results of this study and the study protocol can be read elsewhere [12, 13]. The study was approved by the Medical Research Ethics Committee. All patients gave written informed consent.

Adult patients (aged 18 years or older) with a simple elbow dislocation were recruited from August 25, 2009 until September 18, 2012. Polytraumatized patients, patients with recurrent or open dislocation, additional traumatic injuries of the affected arm, an indication for surgical intervention, impaired elbow function pre-trauma, previous surgery or fractures involving the elbow, or expected problems with maintaining follow-up were excluded.

\section{Randomization and masking}

Patients were randomly assigned to receive early mobilization (early active movements within the limits of pain, started immediately after closed reduction as tolerated) or plaster immobilization (immobilization in a long arm cast for 3 weeks followed by movements within the limits of pain). In both groups, mobilization was supervised by a physical therapist following a guideline that was designed for this study. Further details concerning the randomization procedure and both interventions can be read in the original article and the study protocol $[12,13]$.

\section{Assessments and follow-up}

Data were obtained during out-patient visits at 1,3 and 6 weeks, and at 3, 6, and 12 months after randomization. During these visits, patients completed questionnaires concerning health-related quality of life and a health care consumption questionnaire. This questionnaire included questions on the number of visits to the physical therapist, general practitioner, and medical specialist, admission to hospital, rehabilitation center or nursing home, medication use, and the use of home care. The questionnaire also included questions concerning work absenteeism and resumption. Questionnaire data were supplemented with data from the patients' medical files.

The primary outcome measure for this analysis was total costs, consisting of direct costs (i.e., costs for treatment and intramural care) and indirect costs (i.e., costs for lost production). Secondary outcome measures included the health-related quality of life using the EuroQol-5D (EQ-5D) [14] and Short Form-36 (SF-36), which are both validated [15]. The use of the EQ-5D is recommended for assessing quality of life in trauma patients especially for economic assessments $[16,17]$. The scores for the physical and mental components of the SF-36 were converted to a norm-based score and compared with the norms for the general population of the United States [15]. As there were no significant differences in quality of life scores between the two groups at 1 year, no cost-effectiveness and cost-utility ratio could be calculated. Therefore, a cost-minimization analysis was performed.

\section{Cost measurement}

The total direct and indirect costs of both treatments were analyzed from a societal perspective and included: (1) inhospital care costs which were subdivided into costs for the primary intervention, costs during follow-up, and costs for diagnosis and treatment of adverse events; (2) out of hospital 
care costs for rehabilitation; and (3) indirect costs due to productivity loss. Costs were calculated by multiplying the volumes with the corresponding unit prices (Table 1). Hospital costs for the primary intervention and costs during follow-up consisted of fixed and variable costs. As no patients were admitted to a nursing home or rehabilitation clinic, these costs were zero for all patients. Lost productivity was represented by the hours of work absence.

The costs for use of the operating room included cost for personnel, anesthesia (not including the wage of the anesthesiologist), and overhead costs. An estimation of these costs was made by calculating the means of the fixed cost prices, which were derived from four participating hospitals (one academic and three regional hospitals). Cost prices for other health care resources were derived from the Dutch manual on cost research [18]. Unit costs for all diagnostic procedures were derived from the Dutch Health Care Authority (NZa, Nederlandse Zorgautoriteit). Medication costs were calculated using standard unit prices as described by the CVZ (College voor zorgverzekeringen, Health Care Insurance Board; online available at https://www.medicijnkosten. $\mathrm{nl})$. Indirect costs due to productivity loss were calculated using the friction cost method, which assumes that initial production levels restore after some period of adaption, taking economic circumstances into account [19].

\section{Statistical analysis}

Analyses were performed using the Statistical Package for the Social Sciences (SPSS) version 21 (IBM Corp. Released 2011. IBM SPSS Statistics for Windows, Armonk, NY, USA). The FuncSiE trial was designed to enroll 100 patients. The sample size calculation was performed from a clinical perspective, and is published elsewhere $[12,13]$.

Analysis was by intention to treat and all statistical tests were two-sided. Missing data were not imputed. Chi-squared analysis was used for statistical testing of categorical data. Univariate analysis of continuous data was done using a Mann-Whitney $U$ test (non-parametric data) or a Student's $T$ test (parametric data). $p$ values $<0.05$ were regarded as statistically significant. Accelerated bootstrapping was used for pairwise comparison of the mean differences in all hospital costs, out of hospital costs, indirect costs and total costs between the two treatment groups. The number of replications was chosen to be 1000 .

SF-36 and EQ-5D were repeatedly measured over time, and were compared between treatment groups using linear mixed-effects regression models. These multilevel models included random effects for the intercepts of the regression model and time coefficient of individual patients. Since the outcome measures were not linearly related with time, the time points were entered as factor. The models included fixed effects for treatment group, involvement of the dominant side, and gender. The effect of age was non-significant in all models and age was therefore not included. The interaction between treatment group and time was included in the model to test for differences between the groups over time (i.e., differences in recovery time). For each follow-up moment, the estimated marginal mean of the EQ-5D utility score and the SF-36 physical component summery (PCS) and mental component summery (MCS) scores were computed per treatment group and compared post hoc using a Bonferroni test to correct for multiple testing. Absence of overlap in the $95 \%$ confidence interval around the marginal means was regarded as significant at $p<0.05$.

\section{Results}

Of the hundred patients enrolled, 48 were assigned to early mobilization and 52 to plaster immobilization (Fig. 1). All patients received the allocated treatment. At 1 year followup, complete cost data were available for 99 patients; one patient in the plaster group was lost to follow-up after 6 months. Apart from a relative predominance of patients with an affected dominant side in the early mobilization group, randomization resulted in similar baseline and injury characteristics in the two groups (Table 2).

\section{Quality of life}

No statistically significant differences in health-related quality of life measured with the EQ-5D and SF-36 between the two groups were noted throughout the 1-year followup (Table 3). The EQ-5D was consistently between 0.82 and 0.89 during follow-up. The SF-36 PCS varied between 42 and 53 and the SF-36 MCS varied between 55 and 59 throughout the whole follow-up. Both component summary scores remained within the population norm of $50 \pm 10$ (SD) points, and were independent of treatment.

\section{Health care costs}

Total costs and costs per category are shown in Table 4 and Fig. 2. The mean total costs per patient were $€ 3624$ (95\% confidence interval (CI) 1966-5281) in the early mobilization group versus $€ 7072$ (95\% CI 3444-10,701) in the plaster group. Although early mobilization was $€ 3449$ less expensive than plaster immobilization, this difference was not statistically significant $(p=0.094)$.

The costs for the primary intervention were $€ 551$ (95\% CI 510-591) in the early mobilization group versus $€ 856$ (95\% CI 551-1161) in the plaster immobilization group $(p=0.058)$. Due to the identical, protocolled follow-up, there was no difference in the follow-up costs; $€ 382$ (95\% CI $349-415)$ in the early mobilization group versus $€ 399$ (95\% 
Table 1 Data sources, sources of valuation and unit prices of all cost categories

\begin{tabular}{|c|c|c|c|c|c|}
\hline Cost categories & Unit & $\begin{array}{l}\text { Number of fixed } \\
\text { units for EM/PI }\end{array}$ & Source of data & Source of valuation & Unit price $(€)$ \\
\hline \multicolumn{6}{|l|}{ Hospital costs-primary intervention } \\
\hline Emergency department visit & Visit & $1 / 1$ & Hospital registry & Cost manual & $€ 161.12$ \\
\hline \multicolumn{6}{|l|}{ Radiology/diagnostics } \\
\hline $\mathrm{X}$-ray ${ }^{\mathrm{a}}$ & X-ray & $3 / 3$ & Hospital registry & $\mathrm{NZa}$ & $€ 51.07$ \\
\hline CT scan & CT scan & Variable & Hospital registry & $\mathrm{NZa}$ & $€ 202.14$ \\
\hline MRI & MRI & Variable & Hospital registry & $\mathrm{NZa}$ & $€ 256.79$ \\
\hline Ultrasound & Ultrasound & Variable & Hospital registry & $\mathrm{NZa}$ & $€ 76.64$ \\
\hline Arthrogram & Arthrogram & Variable & Hospital registry & $\mathrm{NZa}$ & $€ 126.86$ \\
\hline Anesthetics/sedation & Case & Variable & Study/hospital registry & $\mathrm{CVZ}$ & $€ 2.00^{\mathrm{b}}$ \\
\hline Plexus block/regional anesthesia & Case & Variable & Study/hospital registry & Hospital data & $€ 82.00$ \\
\hline \multicolumn{6}{|l|}{ Reduction in operating room } \\
\hline Surgeon & Minute & Variable & Study/hospital registry & Cost manual & $€ 2.41^{\mathrm{c}} / € 1.83^{\mathrm{d}}$ \\
\hline Anesthesiologist & Minute & Variable & Hospital registry & Cost manual & $€ 2.41^{\mathrm{c}} / € 1.83^{\mathrm{d}}$ \\
\hline Operating room ${ }^{\mathrm{e}}$ & Minute & Variable & Study/hospital registry & Hospital data & $€ 14.75^{\mathrm{c} / € 11.87^{\mathrm{d}}}$ \\
\hline \multicolumn{6}{|l|}{ Treatment } \\
\hline Plaster & Plaster & $0 / 1$ & Study registry & Hospital data & $€ 127.18$ \\
\hline Plaster change at 1 week & Plaster & $0 / 1$ & Study/hospital registry & Hospital data & $€ 158.60$ \\
\hline Pressure bandage & Pressure bandage & $1 / 0$ & Study registry & $\begin{array}{l}\text { Hospital data and } \\
\text { https://www. } \\
\text { medischservice.nl }\end{array}$ & $€ 15.21$ \\
\hline Sling & Sling & $1 / 1$ & Study registry & $\begin{array}{l}\text { Hospital data and } \\
\text { https://www. } \\
\text { medischservice.nl }\end{array}$ & $€ 15.00$ \\
\hline Admission days & Days & Variable & Study/hospital registry & Cost manual & $€ 464.15^{\mathrm{c}} / € 613.53^{\mathrm{d}}$ \\
\hline Visit out-patient clinic or plaster room ${ }^{\mathrm{f}}$ & Visit & $2 / 2$ & Study/hospital registry & Cost manual & $€ 68.29^{\mathrm{c}} / € 137.64^{\mathrm{d}}$ \\
\hline \multicolumn{6}{|l|}{ Hospital costs-follow-up } \\
\hline Visit out-patient clinic ${ }^{g}$ & Visit & $4 / 4$ & Study/hospital registry & Cost manual & As displayed above \\
\hline Radiology/diagnostics ${ }^{\mathrm{h}}$ & Study & $1 / 1$ & Study/hospital registry & $\mathrm{NZa}$ & As displayed above \\
\hline \multicolumn{6}{|c|}{ Hospital costs—adverse events/revision surgery } \\
\hline Visit out-patient clinic or plaster room ${ }^{\mathrm{i}}$ & Visit & Variable & Study/hospital registry & Cost manual & As displayed above \\
\hline Radiology/diagnostics $^{j}$ & Study & Variable & Study/hospital registry & $\mathrm{NZa}$ & As displayed above \\
\hline Operating room ${ }^{\mathrm{e}}$ & Minutes & Variable & Hospital registry & Hospital data & As displayed above \\
\hline Surgeon & Minutes & Variable & Hospital registry & Cost manual & As displayed above \\
\hline Anesthesiologist & Minutes & Variable & Hospital registry & Cost manual & As displayed above \\
\hline \multicolumn{6}{|l|}{ Type of surgery } \\
\hline Arthrolysis $^{\mathrm{e}}$ & Procedure & Variable & Hospital data & Hospital data & $€ 47.10$ \\
\hline Ulnar nerve release $\mathrm{e}^{\mathrm{e}}$ & Procedure & Variable & Hospital data & Hospital data & $€ 56.91$ \\
\hline Arthroscopy wrist ${ }^{\mathrm{e}}$ & Procedure & Variable & Hospital data & Hospital data & $€ 220.17$ \\
\hline Admission days & Days & Variable & Study/hospital registry & Cost manual & As displayed above \\
\hline \multicolumn{6}{|c|}{ Out of hospital costs-follow-up/rehabilitation } \\
\hline General practitioner & Visits & Variable & Study registry & Cost manual & $€ 29.88$ \\
\hline Physical therapy & Visits & Variable & Study registry & Cost manual & $€ 38.41$ \\
\hline Home care & Hours & Variable & Study registry & Cost manual & $€ 37.35$ \\
\hline \multicolumn{6}{|l|}{ Indirect cost } \\
\hline Work absenteeism males $<35$ years & Hours & Variable & Study registry & Cost manual & $€ 25.00$ \\
\hline Work absenteeism males $\geq 35$ years & Hours & Variable & Study registry & Cost manual & $€ 39.00$ \\
\hline Work absenteeism females $<35$ years & Hours & Variable & Study registry & Cost manual & $€ 24.00$ \\
\hline Work absenteeism females $\geq 35$ years & Hours & Variable & Study registry & Cost manual & $€ 30.00$ \\
\hline
\end{tabular}

Number of units for fixed costs are displayed for early mobilization (EM) and plaster immobilization (PI)

${ }^{a}$ Protocolled radiographs prior to and after reduction and after 1 week 
Table 1 (continued)

${ }^{\mathrm{b}}$ Due to the low costs of medication an estimated average of $€ 2$ was maintained per case of anesthetics/sedation

${ }^{\mathrm{c}}$ General hospital

${ }^{\mathrm{d}}$ Academic hospital

${ }^{\text {e}}$ Protocol cost, only materials (OR use, anesthesiologist and surgeon wages not included) average time for plexus block 15 min. Average operating time for arthrolysis, ulnar nerve release and arthroscopy of the wrist were 240, 190 and 75 min, respectively

${ }^{\mathrm{f}}$ Protocolled visits at 1 and 3 weeks

${ }^{g}$ Protocolled visits at 6 weeks, 3 months, 6 months and 1 year

${ }^{\text {h}}$ Protocolled radiographs at 1 year

${ }^{\mathrm{i}}$ Visits as a result of adverse events

${ }^{\mathrm{j}}$ Diagnostics as a result of adverse events

Fig. 1 Trial flow chart

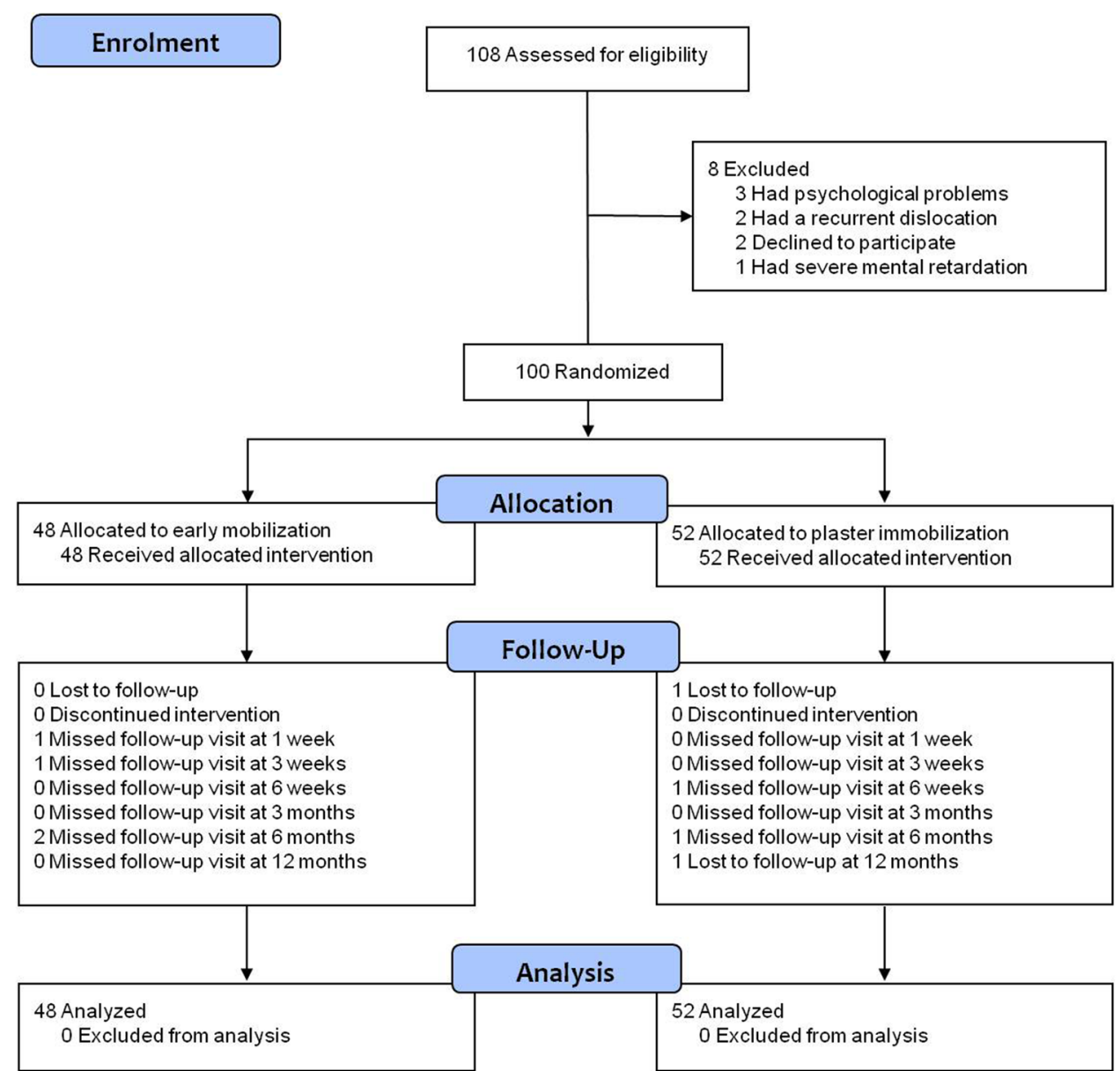

CI 364-434) in the plaster group ( $p=0.481)$. Details concerning adverse events are shown in Table 5. Adverse events occurred in five patients in the early mobilization group versus seven patients in the plaster immobilization group. Costs for diagnosis and treatment of adverse events were $€ 166$ (95\% CI - 147 to 478$)$ in the early mobilization group versus $€ 263$ (95\% CI - 153 to 678) in the plaster immobilization group $(p=0.712)$. The main determinant in the costs for adverse events were costs for surgery. This applied to three patients, one in the early mobilization group (€4744) versus two in the plaster immobilization group ( $€ 3007$ and $€ 1687)$.

The out of hospital costs during follow-up and rehabilitation were $€ 806$ (95\% CI 465-1147) in the early mobilization group versus $€ 966$ (95\% CI 660-1271) in the plaster group ( $p=0.483)$. These costs were mainly due to physical therapy (€738 versus $€ 808$; $p=0.693$; data not shown). This can be explained by the fact that most patients in both groups attended physical therapy to some degree. 
Table 2 Characteristics of trial participants by treatment group

\begin{tabular}{|c|c|c|}
\hline & $\begin{array}{l}\text { Early mobilization } \\
N=48\end{array}$ & $\begin{array}{l}\text { Plaster immobilization } \\
N=52\end{array}$ \\
\hline \multicolumn{3}{|l|}{ Patient characteristics } \\
\hline Male $^{\mathrm{a}}$ & $22(46 \%)$ & $20(39 \%)$ \\
\hline $\mathrm{Age}^{\mathrm{b}}$ (year) & $43(16)$ & $47(14)$ \\
\hline Independent living $^{\mathrm{a}}$ & $44(92 \%)$ & $50(96 \%)$ \\
\hline \multicolumn{3}{|l|}{ Household composition $^{\mathrm{a}}$} \\
\hline Alone & $10(21 \%)$ & $10(19 \%)$ \\
\hline Alone with children & $1(2 \%)$ & $3(6 \%)$ \\
\hline With partner & $18(38 \%)$ & $19(37 \%)$ \\
\hline With partner and children & $13(27 \%)$ & $17(33 \%$ \\
\hline With family/friends & $6(13 \%)$ & $3(6 \%)$ \\
\hline \multicolumn{3}{|l|}{ Activities of daily living } \\
\hline Work participation $(N \text { patients })^{\mathrm{a}}$ & $32(67 \%)$ & $32(62 \%)$ \\
\hline Work participation $(\mathrm{h} / \text { week })^{\mathrm{c}}$ & $36.0(24.0-40.0)$ & $36.0(24.0-40.0)$ \\
\hline \multicolumn{3}{|l|}{ Injury characteristics } \\
\hline Dominant side affected ${ }^{\mathrm{a}}$ & $24(50 \%)$ & $22(42 \%)$ \\
\hline Reduction in operating room ${ }^{a}$ & $5(10 \%)$ & $1(2 \%)$ \\
\hline \multicolumn{3}{|l|}{ Reduction anesthesia ${ }^{\mathrm{a}}$} \\
\hline IV valium & $21(44 \%)$ & $17(33 \%)$ \\
\hline General anesthesia & $10(21 \%)$ & $8(15 \%)$ \\
\hline Intra-articular & $3(6 \%)$ & $12(23 \%)$ \\
\hline None & $6(13 \%)$ & $9(17 \%)$ \\
\hline Other & $6(13 \%)$ & $6(12 \%)$ \\
\hline Regional/plexus & $2(4 \%)$ & $0(0 \%)$ \\
\hline
\end{tabular}

Data are presented as ${ }^{\mathrm{a}} N(\%),{ }^{\mathrm{b}}$ mean $(\mathrm{SD})$, or ${ }^{\mathrm{c}}$ median $\left(P_{25}-P_{75}\right)$

\begin{tabular}{llrr}
\hline Outcome score & Follow-up & Early mobilization & \multicolumn{2}{c}{ Plaster immobilization } \\
& & $N=48$ & \\
\hline EQ-5D utility score & 6 weeks & $0.86(0.83-0.89)$ & $0.82(0.79-0.85)$ \\
& 3 months & $0.87(0.84-0.90)$ & $0.86(0.84-0.89)$ \\
& 6 months & $0.88(0.86-0.91)$ & $0.88(0.85-0.91)$ \\
& 12 months & $0.88(0.85-0.91)$ & $0.89(0.87-0.92)$ \\
SF-36 PCS & 6 weeks & $45(43-48)$ & $42(40-44)$ \\
& 3 months & $52(50-54)$ & $50(48-52)$ \\
& 6 months & $53(50-55)$ & $52(50-54)$ \\
SF-36 MCS & 12 months & $53(51-55)$ & $53(51-55)$ \\
& 6 weeks & $56(54-58)$ & $59(57-61)$ \\
& 3 months & $57(55-59)$ & $57(55-59)$ \\
& 6 months & $57(55-59)$ & $56(54-58)$ \\
& 12 months & $55(53-57)$ & $56(54-58)$ \\
\hline
\end{tabular}

Data are shown as the estimated marginal mean with $95 \%$ confidence interval adjusted for involvement of the dominant side and gender. None of the intervals overlapped indicating no statistical significant difference between the treatment groups

EQ-5D, EuroQoL 5D; SF-36, Short Form-36; PCS, Physical Component Summary score; MCS, Mental Component Summary score 
Table 4 Total costs and costs per cost category by treatment group

\begin{tabular}{|c|c|c|c|c|}
\hline Cost categories & $\begin{array}{l}\text { Early mobilization } \\
N=48\end{array}$ & $\begin{array}{l}\text { Plaster immobilization } \\
N=52\end{array}$ & Difference & $p$ value \\
\hline Direct costs & $€ 1904$ (1303 to 2505$)$ & $€ 2483$ (1822 to 3144$)$ & $-€ 579$ & 0.198 \\
\hline Intramural costs & $€ 1098$ (785 to 1411$)$ & $€ 1517$ (1004 to 2031$)$ & $-€ 419$ & 0.173 \\
\hline Primary intervention & $€ 551$ (510 to 591$)$ & $€ 856$ (551 to 1161$)$ & $-€ 305$ & 0.058 \\
\hline Follow-up & $€ 382$ (349 to 415$)$ & $€ 399$ (364 to 434$)$ & $-€ 17$ & 0.481 \\
\hline Adverse events/revision surgery & $€ 166$ (- 147 to 478$)$ & $€ 263$ (- 153 to 678$)$ & $-€ 97$ & 0.712 \\
\hline Out of hospital costs (FU/rehabilitation) & $€ 806$ (465 to 1147$)$ & $€ 966$ (660 to 1271$)$ & $-€ 160$ & 0.483 \\
\hline Indirect costs (productivity loss) & $€ 1719$ (465 to 2974$)$ & $€ 4589$ (1258 to 7920$)$ & $-€ 2870$ & 0.120 \\
\hline Total & $€ 3624$ (1966 to 5281$)$ & $€ 7072$ (3444 to 10,701$)$ & $-€ 3449$ & 0.094 \\
\hline
\end{tabular}

Data are shown as the mean costs per patient with $95 \%$ confidence interval given between brackets and were analyzed with a regression analysis after bootstrapping

FU, follow-up

Fig. 2 Mean total costs and costs per cost category by treatment group
Table 5 Adverse events and secondary interventions by treatment group

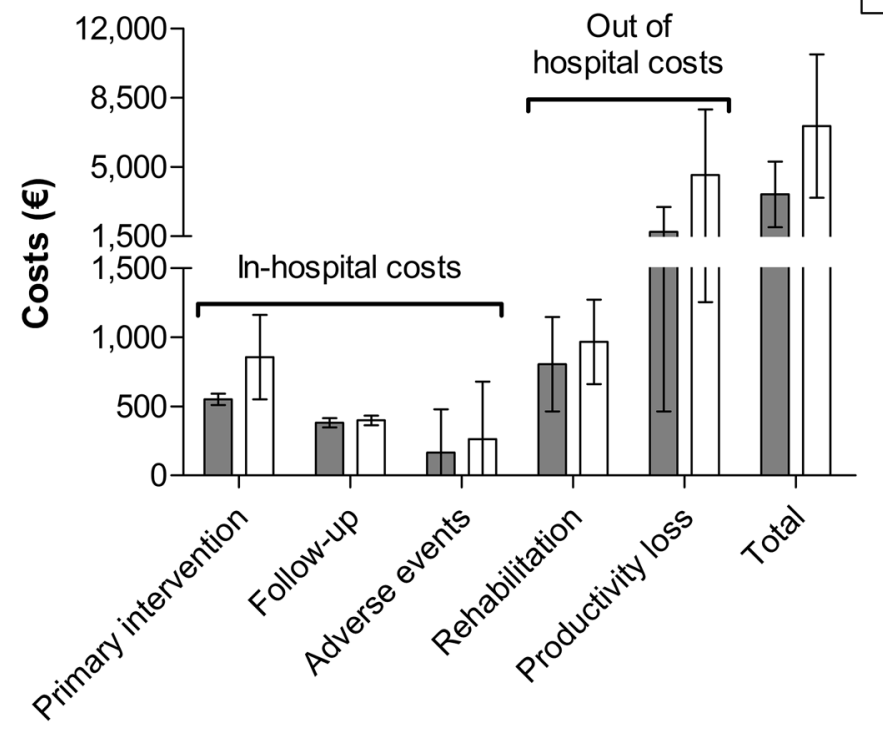

\begin{tabular}{llll}
\hline & $\begin{array}{l}\text { Early } \\
\text { mobilization } \\
N=48\end{array}$ & $\begin{array}{l}\text { Plaster } \\
\text { immobilization } \\
N=52\end{array}$ & $p$ value \\
\hline Adverse events & $5(10 \%)$ & $7(13 \%)$ & 0.640 \\
Secondary interventions $(N$ patients) & $1(2 \%)$ & $2(4 \%)$ & 1.000 \\
Secondary interventions $(N$ interventions) & $1(2 \%)$ & $2(4 \%)$ & 1.000 \\
Arthrolysis & 1 & 0 & \\
Ulnar nerve release & 0 & 1 & \\
Arthroscopy of the wrist & 0 & 1 & \\
\hline
\end{tabular}

Data are presented as $N(\%)$ and were analyzed using a Chi-squared test 
Table 6 Work participation and resumption by treatment group

\begin{tabular}{lccc}
\hline & $\begin{array}{l}\text { Early mobilization } \\
N=48\end{array}$ & $\begin{array}{l}\text { Plaster immobilization } \\
N=52\end{array}$ & $p$ value \\
\hline${\text { Work absenteeism }(N \text { patients })^{\mathrm{A}}}^{\text {Resumption at } 12 \text { months }(N \text { patients })^{\mathrm{A}}}$ & $22(69 \%)$ & $25(78 \%)$ & $0.572^{\mathrm{a}}$ \\
No & $0(0 \%)$ & $1(4 \%)$ & $0.637^{\mathrm{a}}$ \\
Partial & $1(4 \%)$ & $1(4 \%)$ & \\
Fully & $21(96 \%)$ & $23(92 \%)$ & $0.027^{\mathrm{b}}$ \\
$\begin{array}{l}\text { Time of full resumption }(\text { days })^{\mathrm{B}} \\
\text { Hours resumed at } 12 \text { months }(\% \text { of } \\
\text { baseline })^{\mathrm{B}}\end{array}$ & $10(5-16)$ & $18(8-41)$ & $0.376^{\mathrm{b}}$ \\
\hline
\end{tabular}

Data are presented as ${ }^{\mathrm{A}} N(\%)$ or as ${ }^{\mathrm{B}}$ median $\left(P_{25}-P_{75}\right)$ and were analyzed using a ${ }^{\mathrm{a}}$ Chi-squared test and ${ }^{\mathrm{b}}$ Mann-Whitney $U$ test, respectively

\section{Productivity loss}

Work absenteeism did not differ significantly between both groups, although the early mobilization group reported slightly less absenteeism (69\% versus 78\%; $p=0.572$; Table 6). Patients who were treated with early mobilization resumed work 8 days sooner than did patients that were treated with plaster immobilization (10 versus 18 days; $p=0.027)$. The associated mean costs for lost productivity in the total study population were $€ 1719$ (95\% CI 465-2974) in the early mobilization group versus $€ 4589$ (95\% CI 1258-7920) in the plaster group. Despite the large difference of $€ 2870$ in favor of early mobilization, this did not reach statistical significance $(p=0.120)$. When considering only patients that reported sick, the mean costs for productivity loss per absentee were $€ 3751$ (95\% CI 1174-6329) in the early mobilization group and $€ 9546$ (95\% CI 2955-16,137) in the plaster group $(p=0.115)$.

\section{Discussion}

The FuncSiE trial already showed that patients following a simple elbow dislocation demonstrate earlier recovery of elbow function when treated with early mobilization compared with plaster immobilization. As a consequence, early mobilized patients were able to resume work 8 days earlier. Current data demonstrated that health-related quality of life at 1 year was similar in both groups. Early mobilization showed a consistent trend towards being a less expensive treatment than plaster immobilization for all cost categories studied, yet the difference did not reach statistical significance. Surprisingly, there was also no statistically significant difference in costs for physical therapy between the two groups, despite earlier recovery of elbow function in patients that were treated with early mobilization. This could be explained by the fact that both groups received physical therapy according to an identical treatment protocol. Therefore, these data do not allow to reliably answer the questions whether earlier functional recovery after early mobilization consequently leads to less physical therapy in terms of frequency and duration and whether in that way a reduction in care costs might be realized.

\section{Comparison with other studies}

The only recent study on this subject is a retrospective study, which reported the direct healthcare costs of simple elbow dislocations [20]. They divided all patients into three groups according to duration of plaster immobilization (I: $<2$ weeks, $n=26$, II: $2-3$ weeks; $n=27$ and III: > 3 weeks; $n=14)$. They concluded that the length of elbow immobilization did not influence the medical costs. The median direct costs for a simple elbow dislocation in their population were $€ 1375$, whereas this was $€ 1904$ and $€ 2483$ (early mobilization and plaster, respectively) in our study population. Their retrospective study design could explain this difference, as certain cost categories are almost impossible to collect from hospital charts only, which inevitably introduced bias. Another explanation for higher direct costs in our study could be that patients in our study had a protocolled followup period of 1 year. This implied that patients visited the out-patient clinic even when the elbow was considered as completely recovered, leading to costs that would otherwise not have been made. No studies report the indirect costs due to productivity loss as a result of simple elbow dislocations.

\section{Strengths and limitations}

The most important drawback of this study concerns the absence of significance in the substantial differences between costs in both groups. This was mainly caused by the fact that only three patients underwent surgery as a result of adverse events. This led to excessive total costs for these patients compared with patients who healed uneventfully. These outliers caused considerable variation in costs which could falsely have led to the conclusion that the study lacked power. Moreover, the sample size calculation of the trial 
was performed from a clinical perspective rather than for cost calculation purposes. Statistically significant difference in total costs could have been demonstrated provided each treatment group should have encompassed 134 patients ( $\beta=0.8, \alpha=0.05$ and two-sided testing). A larger sample size would possibly have captured additional complications, altering direct and indirect costs associated with the care of simple elbow dislocations.

Unfortunately, these data did not allow to calculate the cost-effectiveness and cost-utility ratio of early mobilization, as there was no statistically significant difference in health-related quality of life at 1-year follow-up between both groups. On the other hand, there is no relevance in performing a cost-effectiveness or cost-utility analysis for a treatment that leads to earlier functional recovery at almost half the costs per patient ( $€ 3449$ less expensive).

A strength of this study is the data completeness. All data were prospectively collected during the entire rehabilitation process, thus giving a truthful reflection of the actual total costs following a simple elbow dislocation. The incidence rate of elbow dislocations in the Netherlands is 5.6 (per 100,000 person years) [2]. The difference of $€ 3449$ in total costs was not statistically significant, but changing treatment protocols for simple elbow dislocations could, in the current Dutch population (16.8 million persons, source: https ://www.cbs.nl/nl-NL/menu/cijfers/default.htm, last update April 2014), reduce the care costs by at least 3.2 million euro per year, supporting the societal relevance of early mobilization.

\section{Conclusion}

The results of this study show that early mobilization of adult patients with a simple elbow dislocation leads to earlier resumption of activities of daily living and work, which might reduce costs by approximately $50 \%$. The results of the FuncSiE trial provided clinical evidence supporting early mobilization after simple elbow dislocations. Current analysis proved that early mobilization should also be the treatment of choice for this injury from a socio-economic point of view.

Acknowledgements Mr. Kiran C. Mahabier, Mr. Harold Goei, Mr. Gerben De Reus and Mrs. Liza Van Loon are acknowledged for their assistance in data collection. Roelf S. Breederveld (Department of Surgery, Red Cross Hospital, Beverwijk, The Netherlands); Maarten W. G. A. Bronkhorst (Department of Surgery, Bronovo Hospital, The Hague, The Netherlands); Jeroen De Haan (Department of Surgery, Westfriesgasthuis, Hoorn, The Netherlands), Mark R. De Vries (Department of Surgery, Reinier de Graaf Gasthuis, Delft, The Netherlands); Boudewijn J. Dwars (Department of Surgery, Slotervaart Hospital, Amsterdam, The Netherlands); Robert Haverlag (Department of Surgery, Onze Lieve Vrouwe Gasthuis, Amsterdam, The Netherlands); Sven A. G. Meylaerts (Department of Surgery, Medical Center
Haaglanden, The Hague, The Netherlands); Jan-Willem R. Mulder (Department of Surgery, Zaans Medical Center, Zaandam, The Netherlands); Peter Patka (Accident and Emergency Department, Erasmus MC, Rotterdam, The Netherlands); Kees Jan Ponsen (Trauma Unit, Department of Surgery, Academic Medical Center, Amsterdam, The Netherlands); W. Herbert Roerdink (Department of Surgery, Deventer Hospital, Deventer, The Netherlands); Gert R. Roukema (Department of Surgery, Maasstad Hospital, Rotterdam, The Netherlands); Inger B. Schipper (Department of Trauma Surgery, Leiden University Medical Center, Leiden, The Netherlands); Michel A. Schouten (Department of Surgery, Hospital Rivierenland, Tiel, The Netherlands); Jan Bernard Sintenie (Department of Surgery, Elkerliek Hospital, Helmond, The Netherlands); Senail Sivro (Department of Surgery, Flevo Hospital, Almere, The Netherlands); Wim E. Tuinebreijer (Trauma Research Unit department of Surgery, Erasmus MC, Rotterdam, The Netherlands); Johan G. H. Van den Brand (Department of Surgery, Medical Center Alkmaar, Alkmaar, The Netherlands); Frits M. Van der Linden (Department of Surgery, Groene Hart Hospital, Gouda, The Netherlands); Hub G. W. M. Van der Meulen (Department of Surgery, Haga Hospital, The Hague, The Netherlands); Egbert J. M. M. Verleisdonk (Department of Surgery, Diakonessenhuis, Utrecht, The Netherlands); Jos P. A. M. Vroemen (Department of Surgery, Amphia Hospital, Breda, The Netherlands); Marco Waleboer (Department of Surgery, Admiraal de Ruyter Hospital, Goes, The Netherlands); W. Jaap Willems (Department of Orthopaedic Surgery, Onze Lieve Vrouwe Gasthuis, Amsterdam, The Netherlands).

Author contributions DDH acted as trial principal investigator. EMMVL, NWLS, and DDH designed the study and trial documents. GITI performed data acquisition. EMMVL, GITI and SP performed the statistical analysis. EMMVL, GITI, and DDH drafted the manuscript. All authors critically reviewed the data, critically revised the manuscript, and read and approved the final manuscript. All site principal investigators (RSB, MWGAB, JDH, MRDV, BJD, RH, SAGM, JWRM, PP, KJP, WHR, GRR, IBS, MAS, JBS, SS, WET, JGHVDB, FMVDL, HGWMVDM, EJMMV, JPAMV, MW and WJW) participated in patient inclusion, critically revised the manuscript, and read and approved the final version.

Funding This project was supported by a grant from the European Society for Surgery of the Shoulder and the Elbow (Fifth SECEC/ ESSSE Research Grant 2010).

\section{Compliance with ethical standards}

Conflict of interest The authors declare that they have no conflict of interest.

Ethical approval The trial is registered at the Netherlands Trial Register (NTR2025). The study was approved by the Medical Research Ethics Committee Erasmus MC, Rotterdam, The Netherlands (Ref. no. MEC-2009-239, NL24128.078.09; approval date 26-SEP-2009).

Informed consent All patients gave written informed consent.

Open Access This article is distributed under the terms of the Creative Commons Attribution 4.0 International License (http://creativeco mmons.org/licenses/by/4.0/), which permits unrestricted use, distribution, and reproduction in any medium, provided you give appropriate credit to the original author(s) and the source, provide a link to the Creative Commons license, and indicate if changes were made. 


\section{References}

1. Josefsson PO, Gentz CF, Johnell O, Wendeberg B (1987) Surgical versus non-surgical treatment of ligamentous injuries following dislocation of the elbow joint. A prospective randomized study. J Bone Jt Surg Am 69(4):605-608

2. Polinder S, Iordens GIT, Panneman MJM, Eygendaal D, Patka P, Den Hartog D et al (2013) Trends in incidence and costs of injuries to the shoulder, arm and wrist in The Netherlands between 1986 and 2008. BMC Public Health 13:531

3. Stoneback JW, Owens BD, Sykes J, Athwal GS, Pointer L, Wolf JM (2012) Incidence of elbow dislocations in the United States population. J Bone Jt Surg Am 94(3):240-245

4. De Haan J, Schep NWL, Tuinebreijer WE, Den Hartog D (2010) Complex and unstable simple elbow dislocations: a review and quantitative analysis of individual patient data. Open Orthop J 4:80-86

5. Anakwe RE, Middleton SD, Jenkins PJ, McQueen MM, CourtBrown CM (2011) Patient-reported outcomes after simple dislocation of the elbow. J Bone Jt Surg Am 93(13):1220-1226

6. De Haan J, Schep NWL, Tuinebreijer WE, Patka P, Den Hartog D (2010) Simple elbow dislocations: a systematic review of the literature. Arch Orthop Trauma Surg 130(2):241-249

7. Maripuri SN, Debnath UK, Rao P, Mohanty K (2007) Simple elbow dislocation among adults: a comparative study of two different methods of treatment. Injury 38(11):1254-1258

8. Mehlhoff TL, Noble PC, Bennett JB, Tullos HS (1988) Simple dislocation of the elbow in the adult. Results after closed treatment. J Bone Jt Surg Am 70(2):244-249

9. Rafai M, Largab A, Cohen D, Trafeh M (1999) Pure posterior luxation of the elbow in adults: immobilization or early mobilization. A randomized prospective study of 50 cases [Luxation posterieure pure du coude chez l'adulte: immobilisation ou mobilisation precoce. Etude prospective randomisee sur $50 \mathrm{cas}]$. Chir Main 18(4):272-278

10. Schippinger G, Seibert FJ, Steinbock J, Kucharczyk M (1999) Management of simple elbow dislocations. Does the period of immobilization affect the eventual results? Langenbecks Arch Surg 384(3):294-297

11. De Haan J, Schep NWL, Peters RW, Tuinebreijer WE, Den Hartog D (2009) Simple elbow dislocations in the Netherlands: what are Dutch surgeons doing? Article in Dutch. Neth J Trauma Surg 17(5):124-127
12. Iordens GIT, Van Lieshout EMM, Schep NWL, De Haan J, Tuinebreijer WE, Eygendaal D et al (2017) Early mobilisation versus plaster immobilisation of simple elbow dislocations: results of the FuncSiE multicentre randomised clinical trial. Br J Sports Med 51(6):531-538

13. De Haan J, Den Hartog D, Tuinebreijer WE, Iordens GIT, Breederveld RS, Bronkhorst MWGA et al (2010) Functional treatment versus plaster for simple elbow dislocations (FuncSiE): a randomized trial. BMC Musculoskelet Disord 11:263

14. Lamers LM, Stalmeier PF, McDonnell J, Krabbe PF, van Busschbach JJ (2005) Measuring the quality of life in economic evaluations: the Dutch EQ-5D tariff [Kwaliteit van leven meten in economische evaluaties: het Nederlands EQ-5D-tarief]. Ned Tijdschr Geneeskd 149(28):1574-1578

15. Ware JE Jr, Sherbourne CD (1992) The MOS 36-item short-form health survey (SF-36). I. Conceptual framework and item selection. Med Care 30(6):473-483

16. Neugebauer E, Bouillon B, Bullinger M, Wood-Dauphinee S (2002) Quality of life after multiple trauma—summary and recommendations of the consensus conference. Restor Neurol Neurosci 20(3-4):161-167

17. Van Beeck EF, Larsen CF, Lyons RA, Meerding WJ, Mulder S, Essink-Bot ML (2007) Guidelines for the conduction of follow-up studies measuring injury-related disability. J Trauma 62(2):534-550

18. Hakkaart-Van Roijen LT (2010) Manual for cost research, methods and standard pricing for economic evaluations in healthcare. Handleiding voor kostenonderzoek, methoden en standaard kostprijzen voor economische evaluaties in de gezondheidszorg: College voor zorgverzekeringen (Geactualiseerde versie 2010)

19. Koopmanschap MA, Rutten FF, van Ineveld BM, van Roijen L (1995) The friction cost method for measuring indirect costs of disease. J Health Econ 14(2):171-189

20. Panteli M, Pountos I, Kanakaris NK, Tosounidis TH, Giannoudis PV (2015) Cost analysis and outcomes of simple elbow dislocations. World J Orthop 6(7):513-520

Publisher's Note Springer Nature remains neutral with regard to jurisdictional claims in published maps and institutional affiliations. 